\title{
Foxo1 is required in mouse spermatogonial stem cells for their maintenance and the initiation of spermatogenesis
}

\author{
Meredith J. Goertz, Zhuoru Wu, Teresa D. Gallardo, F. Kent Hamra, and Diego H. Castrillon \\ Department of Pathology, University of Texas Southwestern Medical Center, Dallas, Texas, USA.
}

\begin{abstract}
Spermatogonial stem cells (SSCs) capable of self-renewal and differentiation are the foundation for spermatogenesis. Although several factors important for these processes have been identified, the fundamental mechanisms regulating SSC self-renewal and differentiation remain unknown. Here, we investigated a role for the Foxo transcription factors in mouse spermatogenesis and found that Foxo1 specifically marks mouse gonocytes and a subset of spermatogonia with stem cell potential. Genetic analyses showed that Foxo1 was required for both SSC homeostasis and the initiation of spermatogenesis. Combined deficiency of Foxo1, Foxo3, and Foxo4 resulted in a severe impairment of SSC self-renewal and a complete block of differentiation, indicating that Foxo3 and Foxo4, although dispensable for male fertility, contribute to SSC function. By conditional inactivation of 3-phosphoinositide-dependent protein kinase 1 (Pdk1) and phosphatase and tensin homolog (Pten) in the male germ line, we found that PI3K signaling regulates Foxo1 stability and subcellular localization, revealing that the Foxos are pivotal effectors of PI3K-Akt signaling in SSCs. We also identified a network of Foxo gene targets - most notably Ret - that rationalized the maintenance of SSCs by the Foxos. These studies demonstrate that Foxo1 expression in the spermatogenic lineage is intimately associated with the stem cell state and revealed what we believe to be novel Foxo-dependent mechanisms underlying SSC selfrenewal and differentiation, with implications for common diseases, including male infertility and testicular cancer, due to abnormalities in SSC function.
\end{abstract}

\section{Introduction}

Spermatogenesis is a dynamic process driven by specialized stem cells - spermatogonia - that in mammals reside on the basement membrane of seminiferous tubules and maintain a single specialized lineage with many cellular intermediates. In addition to a capacity for self-maintenance, $A_{s}$ spermatogonia progress through transit-amplifying intermediates $\left(\mathrm{A}_{\mathrm{pr}}, \mathrm{A}_{\mathrm{al}}, \mathrm{A}_{1-4}\right)$ into $\mathrm{B}$ spermatogonia, which differentiate into spermatocytes that proceed through meiosis to generate round spermatids. An elaborate program of cytoskeletal rearrangement and chromatin condensation (1), known as spermiogenesis, then produces elongated spermatozoa. The concentric geometric arrangement of these cellular intermediates, as spermatogenesis proceeds from the periphery toward the lumen, combined with the ability to identify diverse cell types by simple histologic methods makes the testis an ideal model system for studies of adult stem cell maintenance and differentiation (2). Furthermore, spermatogenesis is not required for life, greatly facilitating the generation of mutants and analysis of phenotypes.

At birth, the testis contains small numbers of specialized and quiescent germ cells termed gonocytes. Postnatally, gonocytes initiate a wave of mitosis and mature to produce an expanded pool of spermatogonial stem cells (SSCs). In the adult, single $\left(A_{s}\right)$ spermatogonia initiate mitotic divisions with incomplete cytokinesis to produce chains of 2-16 spermatogonia $\left(A_{\mathrm{pr}}\right.$ and $\left.\mathrm{A}_{\mathrm{al}}\right)$. The spermatogonia $\left(A_{s}, A_{p r}, A_{a l}\right)$ represented by these steps are termed undifferentiated spermatogonia. $\mathrm{A}_{\mathrm{al}}$ spermatogonia mature into $\mathrm{A}_{1}$ spermatogonia, which undergo 5 additional rounds of mitosis

Conflict of interest: The authors have declared that no conflict of interest exists. Citation for this article: J Clin Invest. 2011;121(9):3456-3466. doi:10.1172/JCI57984. and then form $B$ spermatogonia. The spermatogonia $\left(A_{1}-B\right)$ represented by these latter steps are termed differentiated spermatogonia (2). As spermatogonia were once considered synonymous with SSCs, but recent studies have found that a more expanded subset of undifferentiated spermatogonia $\left(\mathrm{A}_{\mathrm{s}}-\mathrm{A}_{\mathrm{al}}\right)$ can function as SSCs $(3,4)$. The subsequent initiation of spermatogenesis and meiotic entry require a complex interplay of factors, including Kit, which is specifically expressed in differentiated spermatogonia $(5,6)$.

Glial cell line-derived neurotrophic factor (Gdnf) serves essential functions in SSC maintenance. Mutant mice with 1 null Gdnfallele undergo SSC depletion, whereas transgenic males overexpressing Gdnf accumulate undifferentiated spermatogonia (7). Gdnf is produced by Sertoli cells (the somatic cells within seminiferous tubules) and acts through a cell surface receptor heterodimer of Ret and Gfra1, both coexpressed in a subset of undifferentiated spermatogonia (8). Gdnf is the critical growth factor permitting limitless expansion of rodent SSCs in culture, although additional growth factors are also needed (9). These cultures consist of $A_{s}, A_{p r}$, and $\mathrm{A}_{\mathrm{al}}$-like spermatogonia capable of self-renewal and immortal growth and can reconstitute spermatogenesis after engraftment in stem cell transplantation assays (2).

The Foxos function at the interface of cellular growth and organismal longevity (10). They are downstream of growth factor and nutrient stimuli and coordinate various responses, including cell cycle arrest and programmed cell death. Foxo1, Foxo3, and Foxo4 are coordinately regulated by Akt-dependent phosphorylation, which leads to their export from the nucleus to the cytoplasm and, thus, their functional inactivation (11). Foxos promote organismal longevity in invertebrates, and, in humans, single nucleotide polymorphisms are associated with extreme longevity, imply- 
A

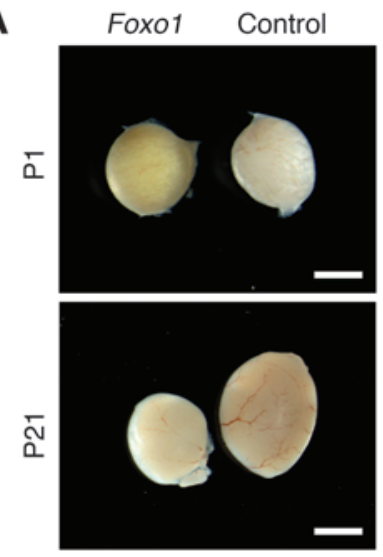

B
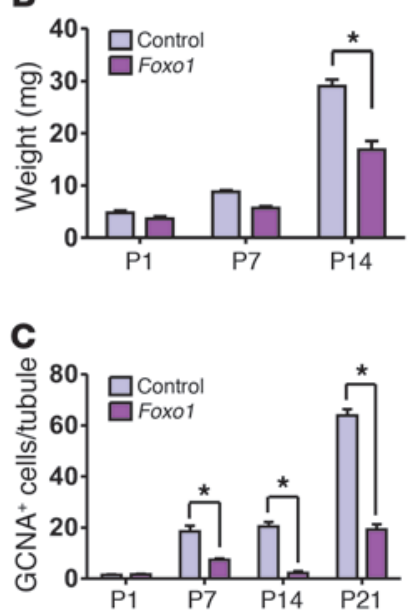

D
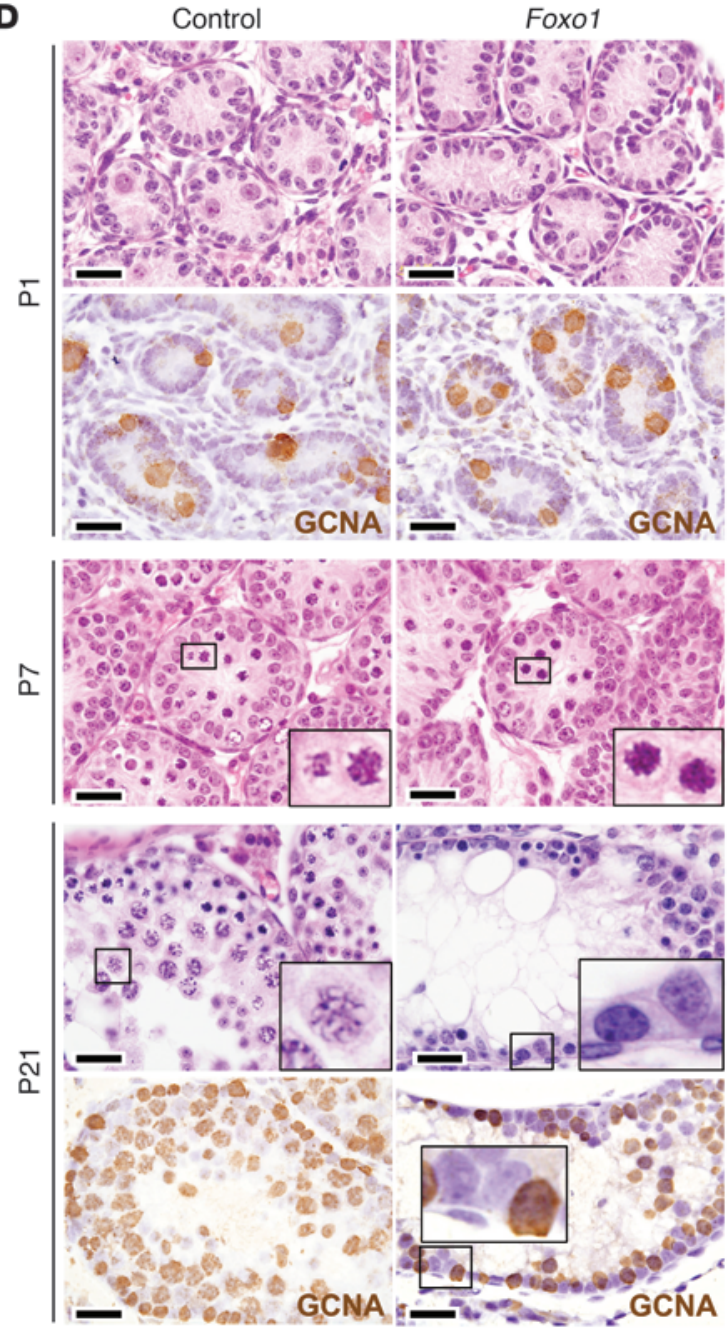

\section{Figure 1}

Foxo1 in SSC maintenance and differentiation in vivo. (A) Intact testes. Scale bar: $3 \mathrm{~mm}$. (B) Testis weights. ${ }^{*} P<0.005$. (C) Germ cells numbers per immunostaining for pan-germ cell marker GCNA. Counts shown are $\mathrm{GCNA}^{+}$cells per tubule. ${ }^{*} P<0.0001$. (D) Testis histology at $\mathrm{P} 1$, $\mathrm{P} 7$, and $\mathrm{P} 21$ in control sibling and Foxo1 mice. For $\mathrm{P} 1$ and $\mathrm{P} 21$, representative GCNA-stained sections are shown. P7 insets show spermatogonia in mitosis. The P21 Foxo1 images are of severely affected tubules. P21 insets show a pachytene spermatocyte (control) or spermatogonia and Sertoli cells (Foxo1). Larger boxes contain high-magnification views of the smaller boxes. Scale bar: $20 \mu \mathrm{m}$. ing important roles in tissue homeostasis and hence adult stem cell function throughout life (12). Consistent with this idea, the Foxos regulate self-renewal in hematopoietic and neural stem cells (13-15). With regards to reproduction, our previous studies in the mouse showed that neither Foxo3 nor Foxo 4 are essential for male fertility, since Foxo3 and Foxo4 nullizygous male mice have normal spermatogenesis, testis weights, and fertility (16-18). In contrast, Foxo1 nullizygosity results in embryonic lethality at approximately E10.5, leaving open the possibility of a unique role of Foxo1 in spermatogenesis $(13,19)$.

On the basis of the role of the Foxos in aging and stem cell function, the cell-type specific nature of Foxo action, and our group's discovery of the role of Foxo3 in female germ line maintenance (16-18), we sought to gain an in-depth understanding of the specific functions of the Foxos in the male germ line, particularly in SSC self-renewal and differentiation. Here, through genetic, cell biological, and gene expression analyses, we demonstrate that among the Foxos, Foxo1 is a unique marker of gonocytes and SSCs in vivo and controls SSC differentiation and self-renewal through a specific network of transcriptional targets, including Ret. We further show through genetic means that PI3K signaling controls both SSC maintenance and differentiation and that the Foxos are pivotal intermediaries of this balance.

\section{Results}

Essential role of Foxo1 in SSC self-renewal and differentiation. To explore the role of the Foxos in spermatogenesis, we first conditionally inactivated Foxo1 (13) in the male germ line with Vasa-cre (also known as Ddx4-cre). Vasa-cre induces recombination in germ cells between E15 and E18 and is expressed in all spermatogenic cells postnatally (20). Vasa-cre Foxo $1^{\text {Lox/Lox }}$ (Vasa-cre Foxo1 ${ }^{L / L}$ ) (hereafter referred to as Foxo1) testes contained normal numbers of gonocytes at P1. However, spermatogonia were reduced by P7, revealing a defect in the proliferative expansion that normally occurs by this time point, and testes were small by P21 (Figure 1). This defect was not due primarily to increased cell death (Supplemental Figure 1A; supplemental material available online with this article; doi:10.1172/JCI57984DS1). Histologic analyses clearly showed distinct defects in SSC self-renewal and differentiation. Most tubules in Foxo1 adults contained spermatogenic cells of diverse states of maturation, albeit at reduced numbers. No spermatozoa were present in adult Foxo1 epididymides, despite the presence of round and elongating spermatids in most tubules (Supplemental Figure 1, B and C), and, as expected given these findings, males were sterile. Some tubules exhibited spermatogonial arrest and failure of meiotic initiation - despite abundant spermatogonia (Figure 1D). Still other Foxo1 tubules 
A
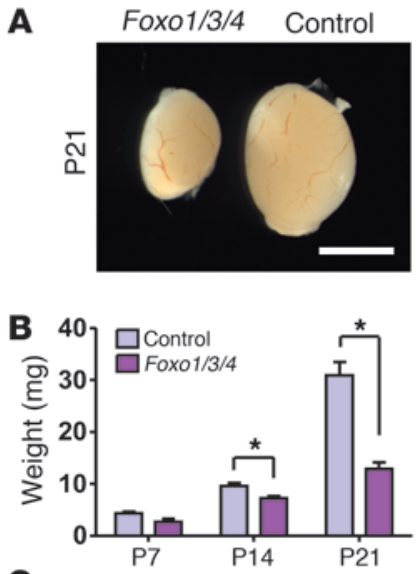

C

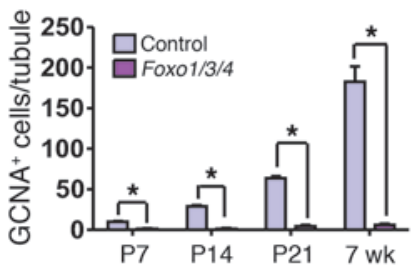

D

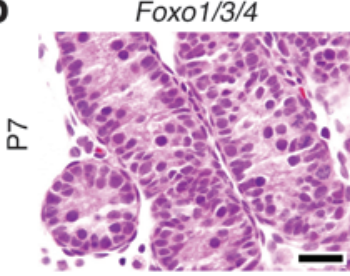

E
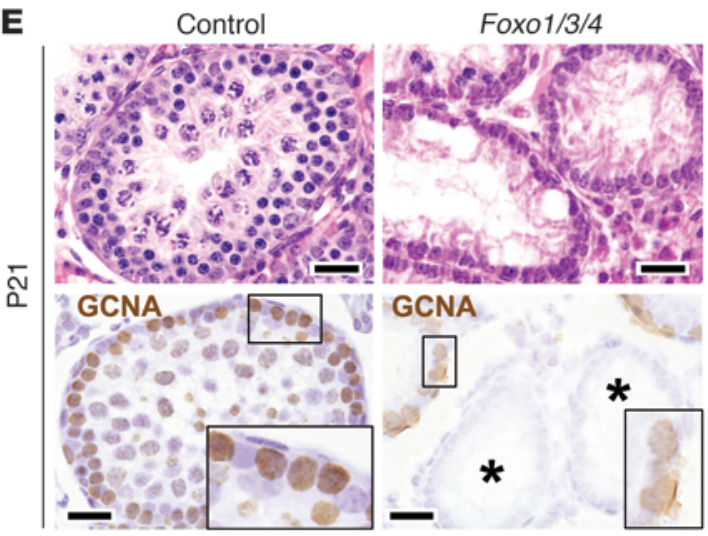

Foxo1/3/4

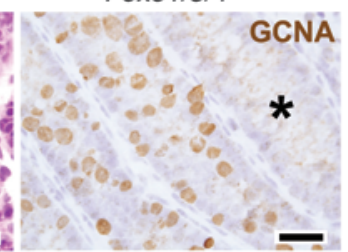
time points. (E) Testis histology for mutant or wild-type controls, H\&E, or immunostains as indicated. Controls have multilayer spermatogenesis, whereas Fox01/3/4 tubules have many spermatogonia (confirmed by GCNA) but no multilayer spermatogenesis. Asterisks indicate empty tubules at P7 and P21. Insets in P21 images highlight basal layer of spermatogonia and complete failure of spermatogenic initiation. Larger boxes contain high-magnification views of the smaller boxes. Scale bar: $20 \mu \mathrm{m}$. were devoid of germ cells (Supplemental Figure 1D), a phenotype never observed in controls, which implies a defect in SSC selfrenewal (21). Older Foxo1 males exhibited similar phenotypes with the exception that empty tubules were not observed (Supplemental Figure 1, D-F). Thus, Foxo1 controls multiple steps of spermatogenesis, from SSC proliferation and self-renewal to the progression of spermatogenesis, including meiosis.

Triple Foxo knockout more clearly exposes Foxo roles in SSC self-renewal and the initiation of spermatogenesis. These genetic analyses demonstrated that Foxo1 is necessary for male fertility and is the most important Foxo in spermatogenesis (since Foxo3 and Foxo4 were previously shown to be dispensable for male fertility) (16-18). However, it remained possible that Foxo3 and Foxo 4 partially compensated for Foxo1 deficiency (13). Vasa-cre Foxo1 ${ }^{L / L}$ Foxo $3^{L / L}$ Foxo $4^{L / L}$ (hereafter referred to as Foxo1/3/4) males had normal gonocyte numbers at birth but exhibited significant testicular hypotrophy by P14 - earlier than that for Foxo1 males, indicating a more severe phenotype (Figure 2, A and B). Compared with those of Foxo1 testes, Foxo1/3/4 testes contained fewer germ cells and more tubules depleted of germ cells at all time points (Figure 2, C-E). It is unclear why some tubules become devoid of germ cells while others do not, but such variation has been documented in other mutants that affect the long-term renewal of SSCs, such as Plzf and Taf4b (21-23). Strikingly, there was also a complete spermatogonial arrest, with failure of spermatogenic differentiation and meiotic initiation evidenced by the absence of multilayer spermatogenesis, including spermatocytes and spermatids in most tubules, even those with abundant spermatogonia (Figure 2E, Supplemental Figure 2, and Supplemental Figure 3A). Thus, Foxo3 and Foxo 4 partially compensate for Foxo 1 in spermatogenesis, and simultaneous inactivation of all 3 Foxos more clearly revealed their collective, essential roles in SSC long-term self-renewal and the initiation of spermatogenesis in the first and subsequent waves of spermatogenesis. Notably, Foxo1/3/4 deficiency phenocopies the failure of spermatogenic initiation and meiotic progression observed in Kit mutants, such as Kit ${ }^{Y 719 F}$, which abrogates signaling through PI3K (24).

Foxo1 is regulated during the gonocyte-SSC transition and is required for the induction of Kit. To begin to understand how Foxo1 controls SSC self-renewal and differentiation, immunolocalization was performed with a Foxo1-specific antibody. Foxo1 was expressed in all gonocytes at P1-P3, while in P21 animals to adult animals, Foxo1 was confined to basal intratubular cells consistent with spermatogonia. Foxo1 was undetectable in the Sertoli cells or any other somatic cell of the testis (P1 to adult) (Figure 3A; see also next section). Strikingly, Foxo1 underwent cytoplasmic-to-nuclear translocation during normal development. At P1, the protein was exclusively cytoplasmic, but nuclear translocation began at P3 and increased by P7. At P3 to P7, the translocation of Foxo 1 protein was not perfectly synchronized, with Foxo1 being distinctly nuclear in some gonocytes but cytoplasmic in others (Figure 3, A and B). The biological basis of this variation is unclear but likely reflects differences in rates of gonocyte maturation, with the gonocytes exhibiting nuclear Foxo1 being more mature. By P21, Foxo1 was nuclear and remained so in adults (Figure 3, A and B, and Supplemental Figure 3B). Thus, we believe Foxo1 is a novel marker of gonocytes, and its cytoplasmic-to-nuclear translocation demarcates the gonocyte-to-SSC transition. Foxo1 testes served as negative controls in these studies, demonstrating the specificity of immunodetection. Note that the complete absence of Foxo $1^{+}$cells in Foxo 1 testes by $\mathrm{P} 7$ proved there was no mosaicism with regards to Vasa-cre-mediated recombination (Figure $3 \mathrm{~A}$ ).

Intriguingly, this translocation of Foxo 1 into the nucleus preceded the normal induction of Kit. Consistent with previous studies (25), Kit was first detectable in wild-type testes at P3, when Foxo1 first entered the nucleus. Kit increased with continued cytoplasmic-tonuclear translocation of Foxo1 (Figure 3, A and B). This induction of Kit protein matched closely with Kit mRNA levels, suggesting that Kit is regulated transcriptionally (Figure 3C). Kit was greatly diminished in Foxo1 and Foxo1/3/4 differentiated spermatogonia 
A

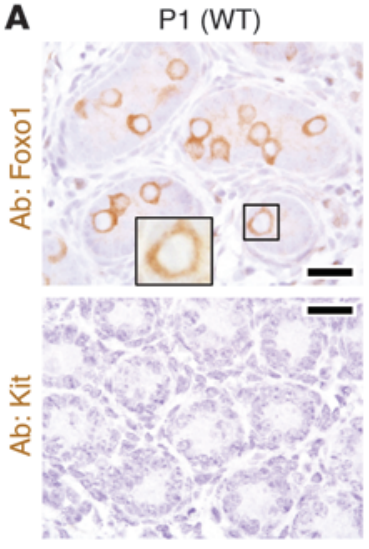

B

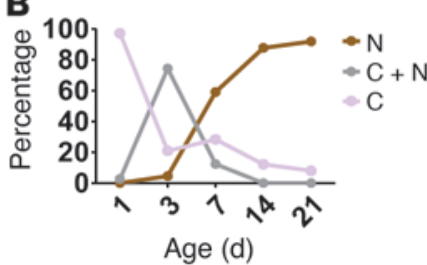

P3 (WT)

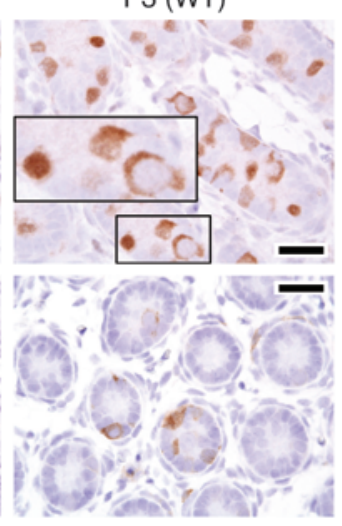

P7 (WT)

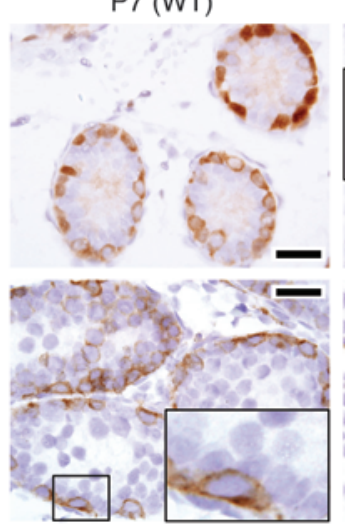

P21 (WT)

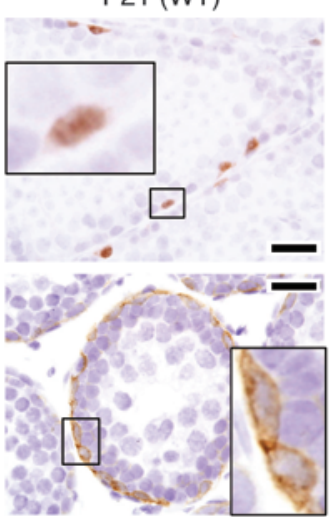

P7 (Fox01)

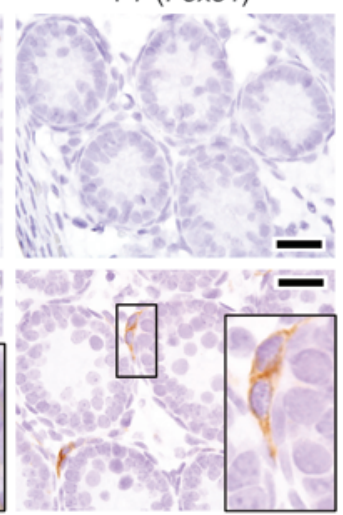

C

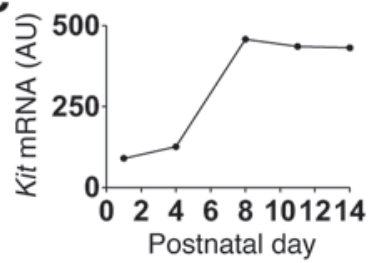

Figure 3

Foxo1 is specifically expressed in gonocytes and undifferentiated spermatogonia and regulates Kit. (A) Kit and Foxo1 immunostains at time points and genotypes as shown. P7 Foxo1 testis serves as negative control for Foxo1 immunostaining. Larger boxes contain high-magnification views of the smaller boxes. The Kit inset shows Kit expression in Leydig cells as internal positive control. Other insets illustrate subcellular distributions of Foxo1 and Kit. Scale bar: $20 \mu \mathrm{m}$. (B) Subcellular distribution of Foxo1 in spermatogonia. C, cytoplasmic; N, nuclear. (C) Developmental profile of Kit mRNA levels in mouse testis by microarray (arbitrary units on $y$ axis).

at P7 and adulthood (Figure 3A and Supplemental Figure 3, D and E). The presence of diverse steps of spermatogenesis in Foxo1 testes argues that Kit underexpression in these testes is not simply explained by the absence of differentiated spermatogonia that normally express Kit. $\mathrm{Plzf}^{+}$(undifferentiated) and $\mathrm{Plzf}^{-}$(differentiated) spermatogonia were present in Foxo1/3/4 testes, further arguing against a maturational block as the basis for Kit underexpression (Supplemental Figure 3C). However, it remains possible that some skipping of developmental steps occurs in mice lacking Foxo gene expression, as has recently been described in another spermatogenesis mutant (Dmrt1) (26). The attenuated induction of Kit in Foxo mutants rationalizes the observed defects in meiosis, given that Kit signaling is vital for this process (24).

The converse possibility that Kit regulates Foxo1 (creating a feedback loop) also appeared plausible, given that Kit can activate PI3K-Akt, which in turn regulates (inactivates) the Foxos. We analyzed mice homozygous for Kit ${ }^{Y 719 F}$, a knockin mutation that abrogates Kit signaling through PI3K. As previously reported, Kit ${ }^{Y 719 F}$ testes were populated by numerous SSCs that failed to differentiate and initiate meiosis (24). However, there was no alteration in the expression or subcellular distribution of Foxo1 in Kit ${ }^{Y 719 F}$ males at P1 to P21 (Supplemental Figure 4). Thus, Foxo1 is required for the induction of Kit expression in differentiating spermatogonia, but the regulation appears unidirectional without evidence of a feedback loop (i.e., Kit is downstream of Foxo1 and acts through signaling intermediaries other than Foxo1 itself).

Foxo1 is a specific marker of undifferentiated spermatogonia in steadystate adult spermatogenesis. Colabeling studies were conducted to further define the spermatogenic cells expressing Foxo1 in the adult testis. In wild-type testes, Foxo 1 and Plzf were always coexpressed
(Figure 4A), demonstrating that, in steady-state spermatogenesis, Foxo 1 expression is restricted to undifferentiated spermatogonia. Next, we studied Gfr $\alpha 1$, which is expressed in single and paired $\left(A_{s}\right.$ and $\left.A_{p r}\right)$ spermatogonia. Only a subset of Foxo $1^{+}$cells were also Gfr $\alpha 1^{+}$, but all Gfr $\alpha 1^{+}$cells were Foxo $1^{+}$, and furthermore, Foxo 1 protein in these cells was predominantly nuclear (Figure 4B). These results are consistent with strong Foxo1 activation in SSCs. The abundance of Foxo1 protein in undifferentiated spermatogonia rationalized the unique genetic requirement for Foxo1 in spermatogenesis, as Foxo3 and Foxo 4 were undetectable immunohistochemically in spermatogonia or any other cell type in testis sections (our unpublished observations). Finally, Foxo1 and Kit were expressed in distinct cells, as expected given that they mark undifferentiated and differentiated spermatogonia, respectively (Figure 4C). Thus, it appears unlikely that Kit is a direct transcriptional target of Foxo1; instead, Foxo 1 must act through some less direct mechanism to regulate Kit expression in progenitor cells. These results are summarized in Figure 4D.

PI3K-Akt signaling operates through the Foxos in the control of SSC homeostasis. Previous studies implicated PI3K-Akt signaling in SSC function $(27,28)$, prompting us to examine whether Foxo1 was an effector of this pathway in spermatogenesis. If so, then phosphatase and tensin homolog (Pten) inactivation should at least partially phenocopy Foxo1/3/4, since Pten inhibits Akt, which in turn inhibits the Foxos (11). Vasa-cre Pten ${ }^{L / L}$ (hereafter referred to as Pten) testes contained normal numbers of gonocytes at P1. There was an initial wave of SSC expansion, as in Foxo1 and Foxo1/3/4 testes; however, severe defects in SSC self-renewal and differentiation became evident later, resulting in testicular hypotrophy and sterility (Figure 5A). Germ cell numbers were normal 

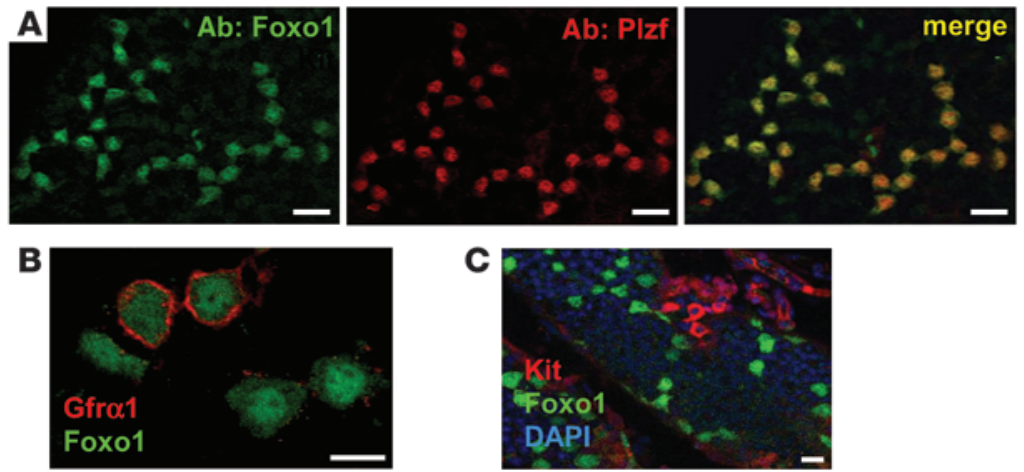

D

Ret $^{+} \mathrm{Grfa} 1^{+}$

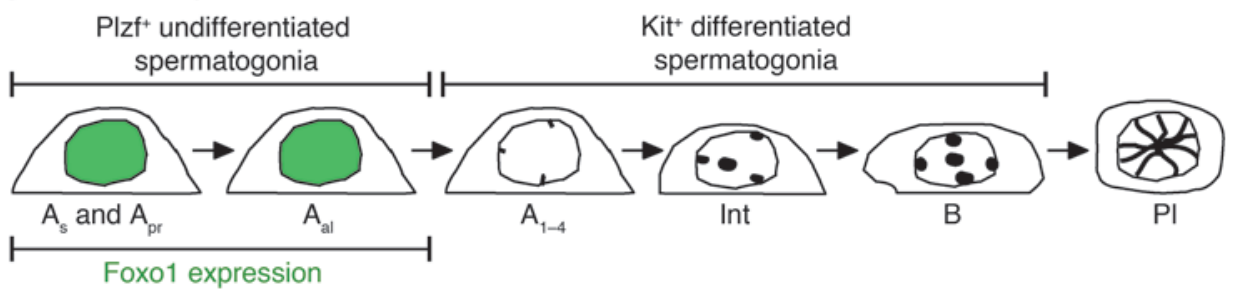

\section{Figure 4}

Foxo1 is specifically expressed in undifferentiated spermatogonia in adult testis. Panels show confocal images of intact tubules. (A) Foxo1 and Plzf coexpression. Shown are two 16-cell clusters. Scale bar: $20 \mu \mathrm{m}$. (B) Gfr 1 and Foxo1 expression. Scale bar: $10 \mu \mathrm{m}$. (C) Kit and Foxo1 nonoverlapping expression. Scale bar: $20 \mu \mathrm{m}$. (D) Schematic illustrating a subset of spermatogonia expressing Foxo1. $A_{s}-B$ spermatogonia to intermediate (Int) and preleptotene (PI) spermatocyte are shown. up to P7 but decreased by P21, and there was a significant agedependent increase in the number of empty tubules (Figure 5, B-D). There was a complete absence of postmeiotic round spermatids (Figure 5D). By P21, no germ cells remained attached to the basement membrane, demonstrating exhaustion of the SSC pool. Testes were almost entirely depleted of germ cells by 4 weeks (Figure 5D). Thus, Pten shares essential roles in SSC self-renewal and spermatogenesis with the Foxos.

Pten loss led to Akt hyperphosphorylation and cytoplasmic localization of Foxo1 by P7, when Foxo1 is normally predominantly nuclear. Based on its weak staining intensity, cytoplasmic Foxo 1 also appeared to be rapidly degraded. This Pten-mediated functional inactivation of Foxo1 was associated with defective Kit induction at P7 (Figure 5E). Thus, Pten acts at least in part through PI3K-Akt and the Foxos to regulate SSC maintenance and differentiation. The more severe phenotype of Pten versus Foxo1/3/4 suggests that additional effectors mediate other actions of PI3K-Akt in the male germ line.

To further investigate the control of the Foxos by the PI3K pathway, we conditionally inactivated 3-phosphoinositide-dependent protein kinase 1 (Pdk1), which is required for Akt activation and thus has a biochemical role opposite to that of Pten. Whereas Pten promotes Akt hyperactivation and Foxo inactivation, Pdk1 loss conversely results in Akt hypoactivation and constitutive Foxo activation (Figure 6A). Vasa-cre $P d k 1^{L / L}$ (hereafter referred to as $P d k 1$ ) males were sterile and their testes were small due to decreased germ cell numbers (Figure 6, B-D). Histologic analyses revealed a complete failure of multilayer spermatogenic differentiation. Notably, the subcellular distribution of Foxo1 was dramatically altered in Pdk1 spermatogonia. Foxo1 was nuclear in virtually all $P d k 1$ spermatogonia at time points in which it is normally cytoplasmic (Figure 6E; $P<0.0001$ at P3 and P14), an effect opposite to that of Pten. Also in sharp contrast to Pten and Foxo, $P d k 1$ testes did not exhibit an age-dependent SSC self-renewal phenotype. Whereas Pten males were depleted of germ cells by 4 weeks, resulting in empty tubules, $P d k 1$ males maintained large numbers of SSCs, which continued to proliferate, as evidenced by abundant $\mathrm{Ki} 67^{+}$positivity. Spermatogonia were numerous, even at 5 months of age, and were Plzf ${ }^{+}$or Plzf-, consistent with undifferentiated and differentiated spermatogonia (Figure 6F). In conclusion, although the signals that regulate Foxo in spermatogonia are not fully known, these findings strongly suggest that they act through PI3K-Akt. Furthermore, our results demonstrate that PI3K-Akt signaling must be carefully titrated in vivo to maintain SSC self-renewal and differentiation and argue that the Foxos are pivotal intermediaries of this balance.

Gene expression analysis identifies Ret and other stem cell factors as Foxo1 targets. Kit underexpression in spermatogonia offered a plausible mechanism for the observed defects in SSC differentiation and meiotic initiation in Foxo mutants. However, our phenotypic analyses also uncovered a discrete Foxo role in SSC self-renewal, a process unaffected by Kit loss of function $(29,30)$. Therefore, Foxo1 must regulate distinct factors relevant to SSC self-renewal. To uncover these, genome-wide transcriptomic analyses were performed on Vasa-cre Foxo $1^{L / L}$ testes versus sibling control Foxo $1^{L / L}$ testes $(n=3$ animals per genotype). $\mathrm{P} 4$ was selected, because at this time point Foxo1 testes were morphologically normal without the cell ratio alterations characterizing later time points. Only 31 genes were differentially expressed per stringent criteria. Foxo1 itself was among the differentially expressed genes, and several genes were represented by nonoverlapping probe sets, suggesting that these targets were physiologically valid. The relatively small number of genes suggests that most may be direct targets (Supplemental Table 1). Quantitative PCR confirmed mRNA level alterations consistent with the microarray data for all loci tested (Figure 7A).

Many of these factors are well-known regulators of stem cell identity in embryonic or adult stem cells, including Gata2, Dppa4, $\underline{\text { Sall }}, \underline{\operatorname{Lhx} 1}$, and Ret $(2,31-34)$, while others are key regulators of spermatogenic progression and meiosis, including Egr2 Egr4, and Tex19 (35-37). That many Foxo1 targets are themselves transcrip- 
A
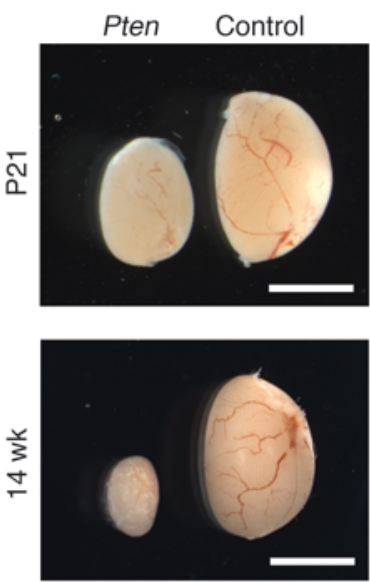

B
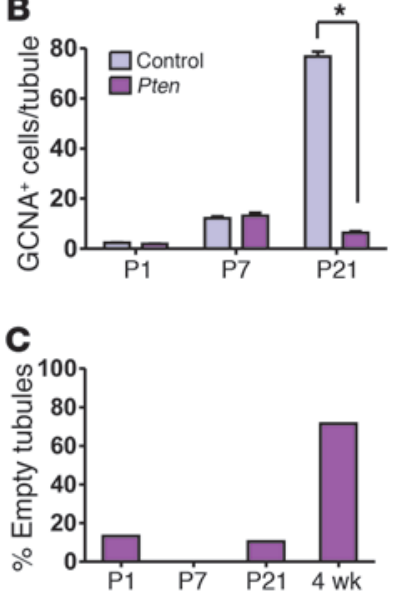

D

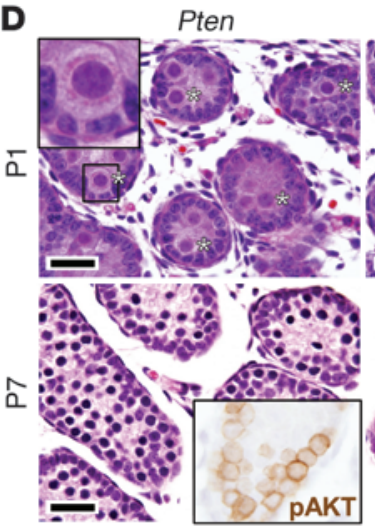
Pten$$
\text { (1) }
$$

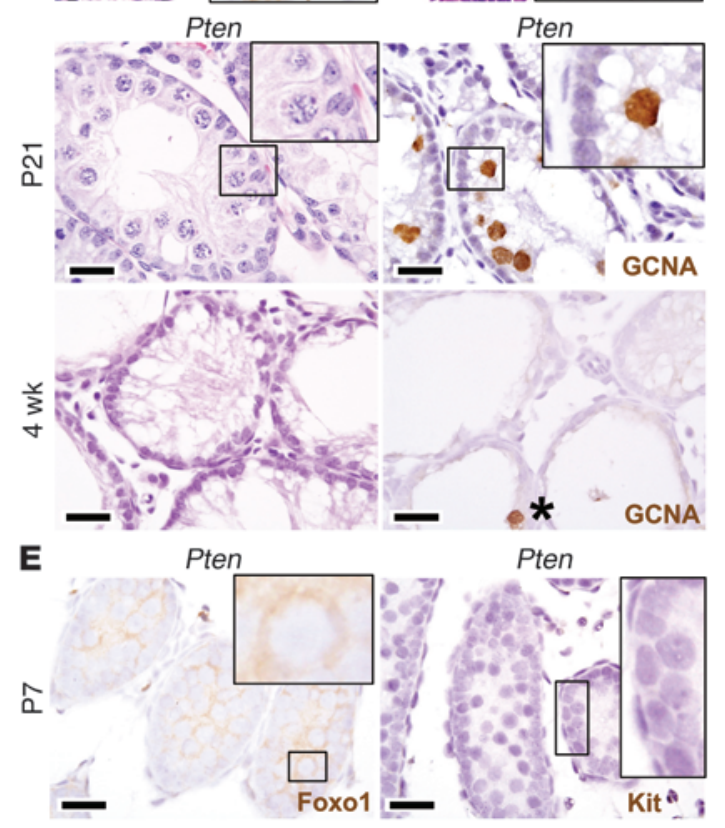

Control

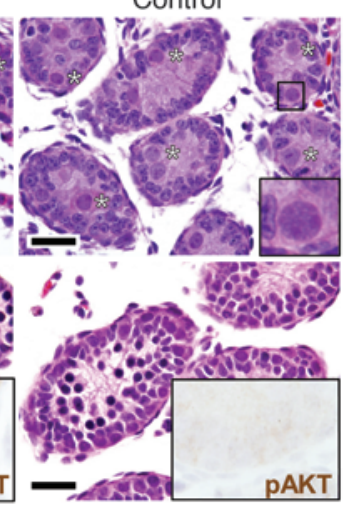

AKT .

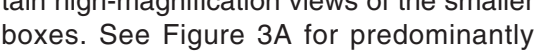
nuclear localization of Foxo1 and expression of Kit in controls.

Figure 5

Regulation of Foxo1 by the PI3K-Akt pathway, Pten, and control testes. (A) Intact testes. Scale bar: $3 \mathrm{~mm}$. (B) GCNA ${ }^{+}$cells per tubule. ${ }^{*} P<0.0001$. (C) Empty tubules harboring no germ cells per GCNA. Control testes contained no empty tubules at these time points. (D) Testis histology, H\&E stains or immunostains as indicated. Asterisks at P1 highlight gonocytes, which were morphologically normal. Asterisk at 4 weeks shows rare residual germ cell. (E) Foxo1 and Kit immunostains of Pten testes at P7. Scale bar: $20 \mu \mathrm{m}$ ( $\mathbf{D}$ and $\mathbf{E})$. Larger boxes contain high-magnification views of the smaller
\end{abstract}

tion factors (underlined) suggests that Foxo1 sits atop a regulatory hierarchy controlling SSC maintenance and differentiation. The identification of Lhx1 is particularly interesting, given that it is directly involved in SSC maintenance (38). Also of note, the Foxo1 transcriptome in male germ cells was distinct from that reported in other cell types, for which genes regulating oxidative stress resistance have been identified as major targets $(14,15,39)$.

Ret appeared to be particularly significant, because its ligand Gdnf is essential for SSC maintenance. In addition, a prior genome-wide screen identified $L b x 1$ and Egr2 among only 6 genes whose expression was strongly Gdnf-dependent in SSCs (40), suggesting we identified a bona fide Gdnf/Ret-associated transcriptional signature. Ret protein was obviously reduced in Foxo1 and Foxo1/3/4 spermatogonia, confirming that Ret expression is regulated by Foxo1 (either directly or indirectly) in vivo (Figure 7B). To further explore the physiologic significance of these findings, Ret was analyzed in Pten and Pdk1 testes, in which the Foxos are constitutively inactivated or activated, respectively. Similar to that in Foxo testes, and as expected, Ret was significantly reduced in Pten testes. However, Ret was strikingly elevated in Pdk1 testes (Figure 7C). These findings strongly argue that the PI3K pathway regulates Ret expression in SSCs and, furthermore, that this regulation occurs via the Foxos. Given the requirement for Ret in
SSC maintenance (Ret loss-of-function mutations result in germ cell depletion in mice), the control of Ret by the PI3K pathway via Foxo 1 rationalizes the SSC self-renewal phenotype observed in our Foxo mutants (41).

\section{Discussion}

This study demonstrates that the Foxos, particularly Foxo1, served critical and nonredundant roles in SSC homeostasis both in vitro and in vivo. In their absence, SSCs displayed a decreased ability to self-renew and differentiate. Foxo function in the male and female germ line shares some similarities. First, the Foxos are not required for the differentiation or early development of the germ lineage in males or females. Rather, the Foxos evolved to control gametogenesis within the gonad itself. Whereas Foxo1 is highly expressed in undifferentiated spermatogonia, Foxo3 is highly expressed in primordial oocytes, in which it serves to restrain their growth (18). The unique genetic requirement for Foxo1 in males and Foxo3 in females mirrors their high expression at discrete cellular stages in spermatogenesis or oogenesis. Another intriguing similarity is that both Foxo1 and Foxo3 undergo relocalization from the cytoplasm to the nucleus postnatally, coincident with their initial functional requirement, suggesting that the timing of their activation is critical for normal germ line development and function. In mammals, 
A
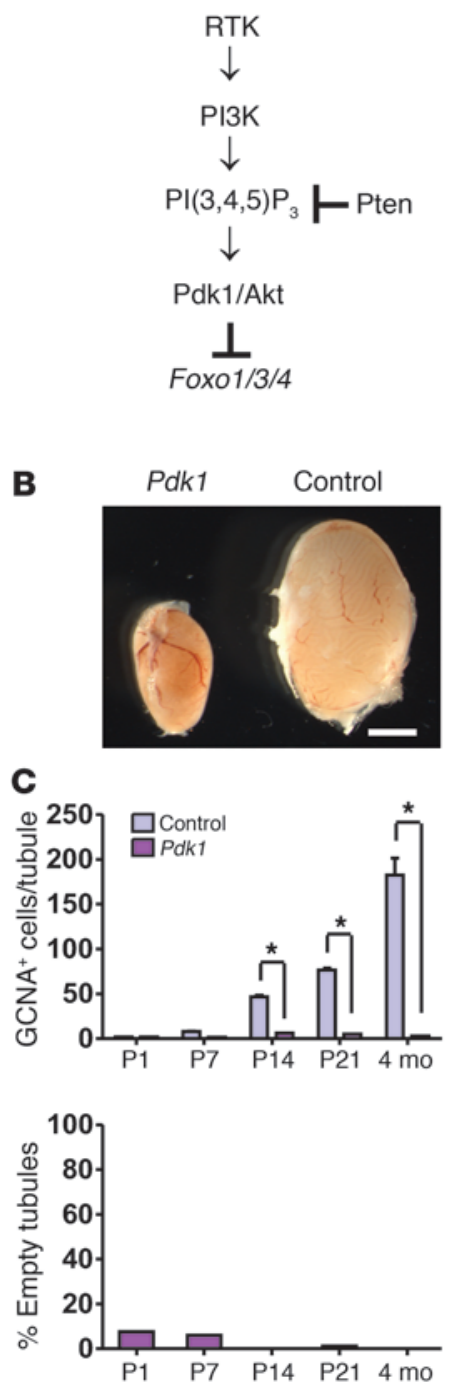

D
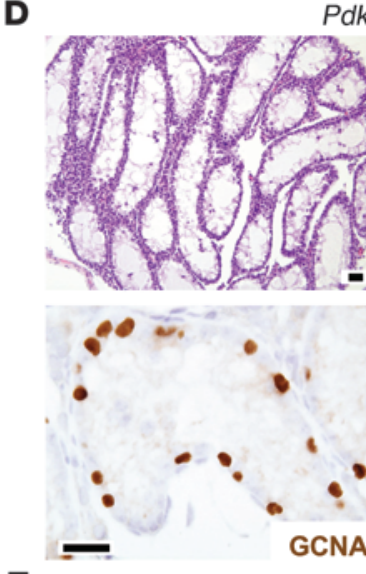

E

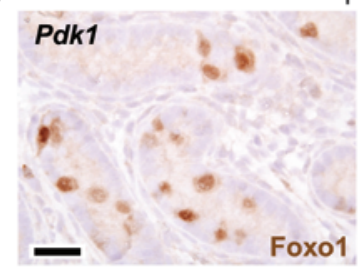

P21

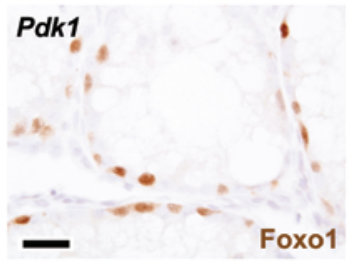

$\mathbf{F}$

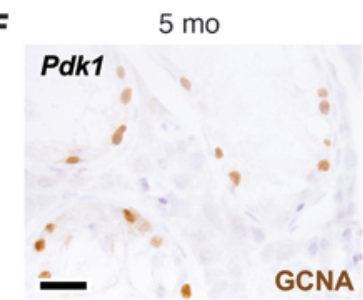

Pdk1 P21

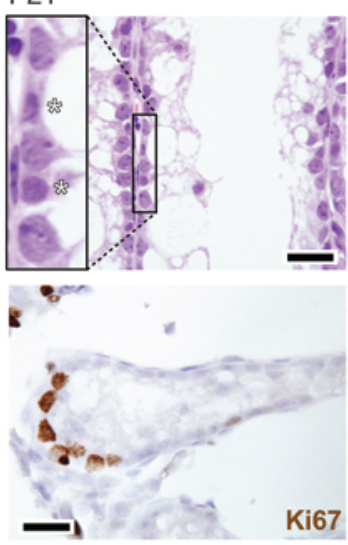

P3

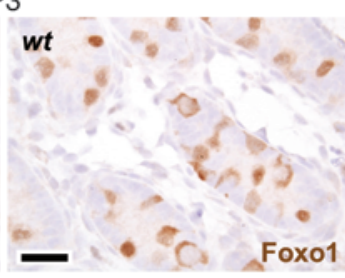

Cytoplasmic Foxo1

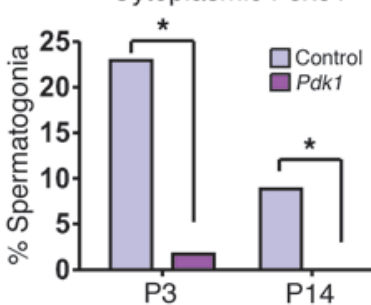

P3

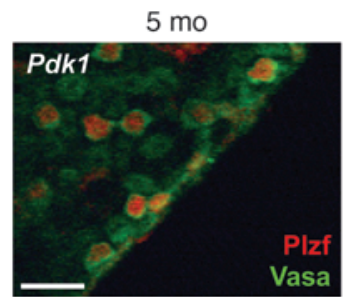

Figure 6

Regulation of Foxo1 by the PI3K-Akt pathway, Pdk1, and control testes. (A) Schematic of the PI3K-Akt-Foxo pathway. A receptor (typically a receptor tyrosine kinase [RTK]) results in PI3K activation and synthesis of 3-phosphoinositides (PIP3). Pten metabolizes PIP3 and acts as a pathway inhibitor. (B) Pdk1 testes were small by P14. Testes from 4-month-old males are shown. Scale bar: $3 \mathrm{~mm}$. (C) $\mathrm{GCNA}^{+}$cells per tubule and tubules devoid of germ cells per GCNA. ${ }^{*} P<0.0001$. (D) Testis histology (H\&E) and immunohistochemistry with antibodies as shown. Inset shows alternating Sertoli cells and spermatogonia (asterisks) on basement membrane with complete absence of differentiation and multilayer spermatogenesis. Ki67 demonstrates that spermatogonia are actively proliferating; the Ki67 index in spermatogonia was more than $10 \%$ (data not shown). (E) Foxo1 localization in Pdk1 testes at various ages as shown. Pdk1 inactivation promotes the shift of Foxo1 protein from cytoplasm to nucleus at P3. ${ }^{*} P<0.0001$. (F) Persistence of SSCs in aged (5-monthold) males. GCNA shows abundant spermatogonia and absence of empty tubules. Plzf and Vasa staining of intact tubules (confocal microscopy) shows the presence of both undifferentiated $\left(\mathrm{Plzf}^{+}\right)$and differentiated $(\mathrm{Plzf})$ Vasa ${ }^{+}$ spermatogonia in aged $P d k 1$ testes. Scale bar: $20 \mu \mathrm{m}$ (D-F). there are 3 principal Foxo genes (and 1 more distantly related member, Foxo6), but there is a single ancestral homolog in Caenorbabditis elegans, daf-16, which controls life span and stress resistance (12). Taken together, studies from our laboratory suggest that this gene duplication conferred an evolutionary advantage by permitting the Foxos to adopt distinct but controlling roles in oogenesis and spermatogenesis, 2 processes with many similarities but also important differences.

One of the more interesting and potentially unexpected findings in this study is that Foxo1 controlled various aspects of spermatogenesis, from long-term SSC self-renewal to the initiation of spermatogenesis and meiosis. The Foxo1 phenotype also indicated a requirement in spermiogenesis. The control of diverse steps of spermatogenesis is surprising, because Foxo 1 protein is restricted to very early progenitors (undifferentiated spermatogonia). We conclude that in addition to regulating the expression of a network of factors required for spermatogenesis, Foxo 1 initiates a cascade of events that influence later steps of spermatogenesis. It is also possible that Foxos are biologically active and regulate gene expression in subsequent steps of spermatogenesis, even if individual Foxos are much less abundant than Foxo1 in undifferentiated spermatogonia. The underexpression of Kit in Foxo and Pten mutants explains the failure of meiotic initiation and represents a molecular foothold in understanding this cascade. However, further investigations will be needed to understand how the individual Foxo targets identified in this study contribute to the diverse steps of spermatogenesis. The Foxos join a growing network of transcription factors - Plzf and Taf $4 b$ - that control early steps of spermatogenesis, including SSC self-renewal (21, 22). Further dissection of functional interrelationships among these factors and their order of interactions is warranted. In particular, additional studies such as ChIP-seq will be needed to formally distinguish between indirect and direct Foxo targets, ascertain whether the Foxos act directly upon the Ret promoter or via 

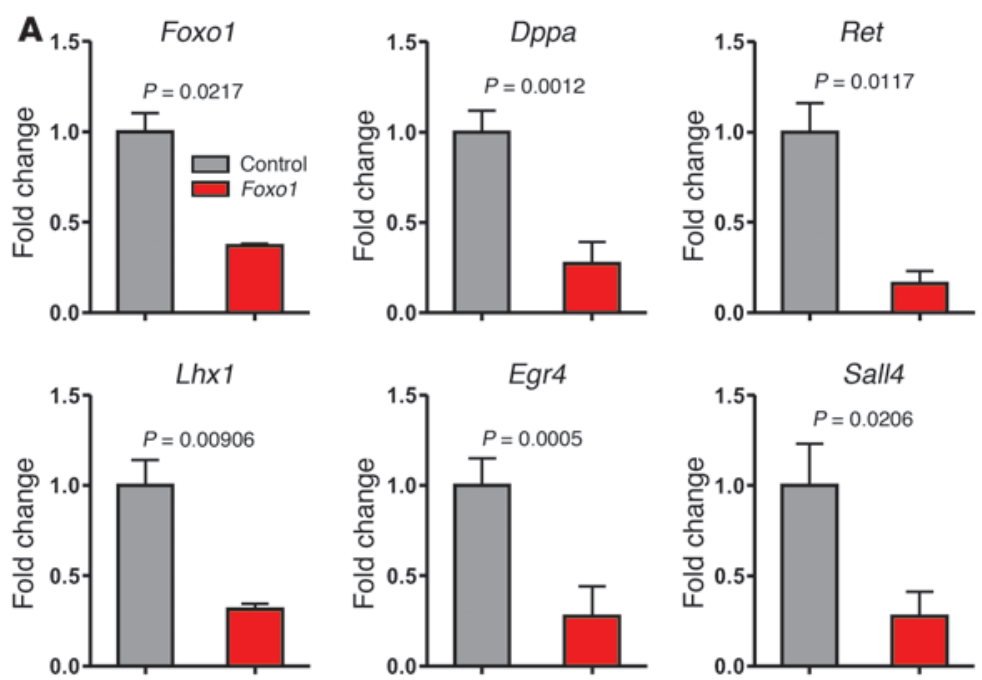

B

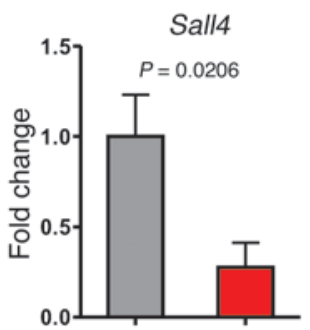

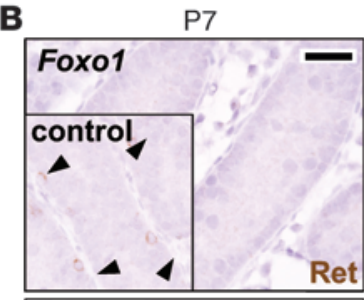
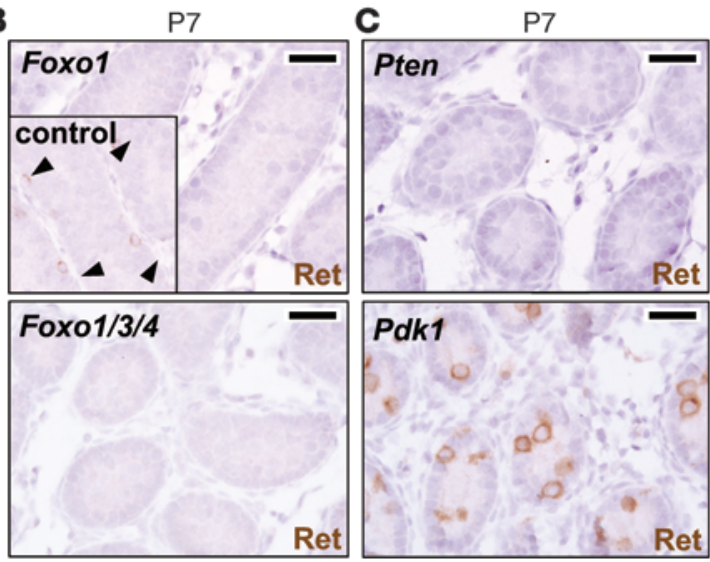

Figure 7

In vivo transcriptomic analysis identifies Foxo1 targets including Ret. (A) Relative mRNA levels in control testes versus Foxo 1 testes $(n=3$ biological replicates) by qPCR. (B) Decreased Ret protein in Foxo1 and Foxo1/3/4 testes by immunohistochemistry; slides were counterstained with hematoxylin. In control testis (inset, at the same magnification), Ret is detectable on cell surface of a subset of undifferentiated spermatogonia (arrows). (C) Ret is decreased in Pten but increased in Pdk1 testes. Scale bar: $20 \mu \mathrm{m}$ (B and C).

other intermediaries, and also determine whether the 3 Foxos act upon distinct direct targets.

The developmental relocalization of Foxo1 during the gonocyte-SSC transition implies that the subcellular localization of the Foxos is an important determinant of their biological activity, consistent with prior observations $(11,18)$. Our studies of Pdk1 and Pten mutants showed that the PI3K-Akt pathway is the principal pathway regulating Foxo1 subcellular localization and activity in the context of spermatogenesis. Foxo1 is highly expressed and in an active (nuclear) state in SSCs and is intimately linked with the "stem state" in spermatogonia. For example, in Pten testes, Foxo1 was inactivated, and SSCs became depleted. In contrast, in Pdk1 testes, Foxo1 remained activated (nuclear), and SSCs were not depleted. These findings also imply that the inactivation of Foxo1 that occurs physiologically triggers the initiation of spermatogenesis. What, in turn, are the extracellular signals influencing this delicate balance of PI3K and hence Foxo 1 activity? Candidates are a variety of growth factor ligands that bind cognate receptor tyrosine kinases or $G$ protein-coupled receptors that act via PI3K. Our studies of the Kit ${ }^{Y 719 F}$ mutant argue that Kit ligand is not critical, even though Kit reexpression coincided with Foxo1 relocalization. In addition, the nuclear and active state of Foxo 1 in $\mathrm{Gfr} \alpha 1^{+} \mathrm{A}_{\mathrm{s}}$ and $A_{p r}$ spermatogonia further argues that the Gdnf-Gfro1/Ret signaling axis is not the principal regulator of Foxo activity in vivo (if that were the case, pathway activity would drive Foxo1 out of the nucleus), although this question merits further exploration.

We demonstrated essential roles for Pdk1 and Pten in the male germ line. Interestingly, both factors are required for spermatogenic differentiation, as evidenced by the absence of multilayer spermatogenesis. Pten inactivation resulted in the extinction of SSCs even before the onset of sexual maturity, whereas in Pdk1 testes, SSCs continued to proliferate in aged males. These results show that SSC maintenance and differentiation depend critically on the proper balance of PI3K-Akt activity and suggest a model in which excessive PI3K activity promotes excessive stem cell loss (see below). The Pdk1 phenotype also suggests that PI3K hypoactivity leads to a failure of spermatogenic progression and meiotic initiation. Although interpretation of these phenotypes may be complicated by the diverse functions of this pathway at multiple points of spermatogenesis, our results strongly argue that the Foxos and their targets are pivotal mediators of this balance.

Our results seem at odds with a report that Pten inactivation led to enhanced germ cell production and testicular teratomas (42). In the prior study, conditional Pten deletion was performed with TNAP-cre, which is induced in primordial germ cells by E13.5, earlier than that with Vasa-cre. All TNAP-cre Pten males developed multiple bilateral teratomas by birth, whereas we did not observe teratomas or any other type of abnormal cellular proliferation. It is possible that the somewhat earlier timing of Pten deletion promoted teratoma formation. Our results, which seem paradoxical given the role of Pten as a cell growth and tumor suppressor, are more in line with those from studies of Pten in HSCs, in which conditional inactivation resulted in abnormalities in differentiation and longterm decline of HSCs due to decreased self-renewal potential (43, 44). These HSC phenotypes can be partially rescued by rapamycin, an mTOR inhibitor. Plzf, a transcription factor needed for SSC maintenance, indirectly regulates mTOR activity (45). Thus, it will be interesting to study the relative contributions and interactions between PI3K-Akt-Foxo and the mTOR pathways in SSCs.

Recent studies have implicated the Foxos in stem cell maintenance. The Foxos, particularly Foxo3, coordinately regulate neural stem cell homeostasis through genes influencing stress responses and oxygen metabolism $(15,39)$. The Foxos also regulate HSC differentiation and assist long-term maintenance by protecting against oxidative stress. In contrast to these studies, our transcriptomic analyses did not yield targets relevant to oxygen metabolism or cellular stress responses. Instead, the Foxos appeared to control a network of genes unique to spermatogenesis, consistent with diverse studies showing that Foxo functions are biologically numerous and highly context-dependent (10). 


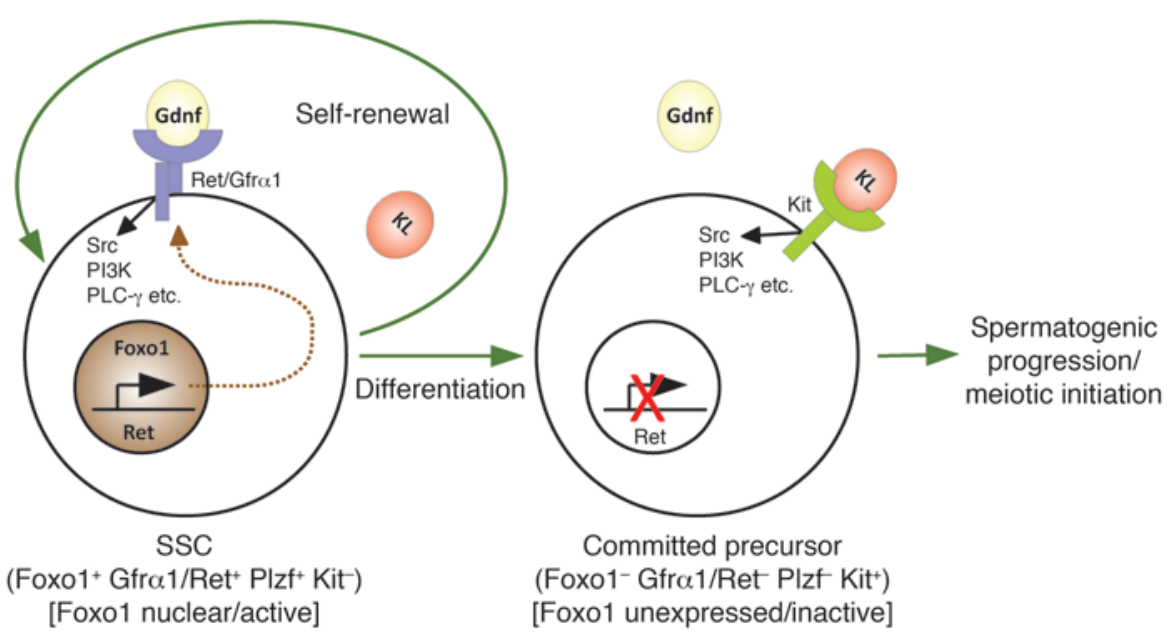

Figure 8

Model for essential role of Foxos in control of SSC self-maintenance and initiation of spermatogenesis. Shown is a SSC capable of self-renewal or commitment to differentiation. Foxo1 is expressed and in an active (nuclear) state in SSCs (brown), promoting transcription of Ret mRNA and high levels of protein on the cell surface. Upon binding to Gdnf, an essential SSC growth factor, Ret/ Gfra1 receptor (blue) signals via diverse signaling pathways to promote SSC survival and selfrenewal. Through mechanisms that are not understood but likely to be PI3K-Akt-dependent (data not shown), Foxo1 becomes inactivated and degraded. Loss of Foxo1 activity then leads to Ret downregulation and commitment to spermatogenic differentiation. This study showed that in addition to serving as a marker for SSCs, Foxo1 is functionally associated with the stem cell state through the regulation of diverse targets, including Ret. For example, after Pten ablation, Foxo1 was inactivated, and Ret expression was low. In contrast, Pdk1 ablation led to constitutive activation of Foxo1 and high Ret expression, promoting continued SSC self-renewal. These findings also imply that PI3K signaling must be carefully titrated to balance between SSC self-renewal and differentiation via the Foxos. Among outstanding remaining questions are (a) how the sequentia expression of the Ret/Gfra1 and Kit receptors is coordinated and regulated directly or indirectly by Foxo1, (b) how these receptors exert differential responses (given that they signal through similar signaling intermediaries), and (c) how other well-known SSC maintenance and differentiation factors, such as PIzf and retinoic acid interact, with PI3K-Foxo. KL, Kit ligand.

The regulation of Ret rationalizes to a large extent the observed Foxo defects in long-term SSC maintenance. By in situ methods, Ret was virtually undetectable in Foxo1 and Foxo1/3/4 undifferentiated spermatogonia in vivo. In enteric neuron progenitors, Ret acts through diverse pathways, including Ras-MAPK, PI3K-Akt, PLC- $\gamma$, and Src pathways, to regulate proliferation, differentiation, and long-term survival (46). Ret also acts through these pathways to promote SSC survival and proliferation $(2,38)$. Ret inactivation results in severe defects in SSC proliferation and differentiation by P7, eventually leading to SSC depletion $(8,41)$. The similarity of the Ret and Foxo phenotypes and these previous studies together strongly argue that Ret downregulation accounts for the observed Foxo phenotypes in SSC maintenance. Finally, the possibility that Foxos control Ret expression in diverse neuronal populations also merits further investigation, particularly as the Foxos promote neuronal survival (47).

The studies presented here, which were based on in vivo analyses of diverse genetic models, including conditional germ line mutants, imply an intimate relationship between Foxo1 expression and stem cell potential. They also argue that the Foxos function in a cellautonomous manner to maintain SSC potential. Future dissection of the role of the Foxos and the PI3K pathway in the regulation of SSC long-term maintenance would likely benefit from the development and exploitation of in vitro models. One such approach would entail the derivation and culture of SSCs that are null or conditionally null for the Foxos or other PI3K pathway components. Such in vitro models, perhaps coupled with transplantation experiments, would enable a diversity of other studies, such as cell cycle analyses, that would likely lead to further insights into the diverse molecular mechanisms by which the PI3K-Foxo pathway regulates the long-term maintenance and differentiation of SSCs.

In conclusion, the Foxos are pivotal regulators of SSC self-renewal and differentiation (summarized in Figure 8). Like other adult stem cells, SSCs represent a finite reserve that needs to be maintained throughout life. The importance of the Foxos in organismal aging (12), combined with our discovery of their function in SSC self-renewal suggests that the Foxos function throughout life to protect this finite resource. It seems likely that abnormalities in SSC maintenance account for some cases of male infertility, one of the most common conditions for which medical attention is sought. Conversely, the overexpansion of cells with stem cell-like properties is thought to be a requisite for cancer. Testicular cancer is the most common cancer in young men, and most of these tumors are composed of cells with SSC-like properties (48). Thus, these findings and future investigations of the PI3K-Akt-Foxo pathway in SSCs using the models described herein should lead to important insights into the etiology of these clinically important but poorly understood conditions.

\section{Methods}

Mouse strains, breeding, and analysis. This study was approved by the University of Texas Southwestern (UTSW) Institutional Animal Care and Use Committee. All alleles were in an $\mathrm{FVB} / \mathrm{n}$ background (backcrossed at least $n=10$ generations). Generation of the Vasa-cre, Pten ${ }^{L / L}, P d k 1^{L / L}, K i t^{Y 719 F}$, and Foxo $1^{L / L} / F_{0 x 0} 3^{L / L} /$ Foxo $4^{L / L}$ alleles and genotyping protocols were previously described (18, 20, 24, 49, 50). Young Vasa-cre males ( $<10$ weeks of age) were used for second-generation cross to avoid potent maternal effects observed with females $(18,20)$ and global recombination sometimes observed with older males. Testes from experimental and control animals $(n=3)$ (siblings not inheriting Vasa-cre) were evaluated as biological replicates for each time point in tissue-based analyses.

Tissue processing, immunohistochemistry, and immunofluorescence. For immunohistochemistry, tissues were fixed in $10 \%$ buffered formalin overnight, embedded in paraffin, and cut into $5-\mu \mathrm{m}$ sections. Slides were deparaffinized in xylene, hydrated in an ethanol series, subjected to antigen retrieval by boiling in $10 \mathrm{mM}$ sodium citrate, and cooled at room temperature (RT) for 20 minutes. The detection system was Immpress (Vector Laboratories). Tissue sections from experimental and control samples were placed on the same slide to ensure identical processing. For whole-mount immunofluorescence, seminiferous tubules were mechanically dissociated in PBS on ice and fixed 
overnight in $4 \%$ paraformaldehyde. Tubules were dehydrated in a series of methanol washes and stored at $-20^{\circ} \mathrm{C}$. To rehydrate and permeabilize, tubules were put through a series of washes with methanol/PBS plus $0.1 \%$ Tween-20. Tubules were blocked with 1\% BSA/PBS at RT (Thermo Scientific Blocker no. 37525) for 2 hours, and primary antibody was added in $0.5 \% \mathrm{BSA} / \mathrm{PBS}$ with $0.02 \%$ sodium azide and incubated at $4{ }^{\circ} \mathrm{C}$ overnight. Tubules were washed 3 times for 10 minutes each time in PBS at RT. Secondary antibody (Invitrogen; Alexa Fluor $555 \alpha$-mouse, catalog no. A-21422, or Alexa Fluor $488 \alpha$-rabbit, catalog no. A-11008) was added at 1:1,000 in $0.5 \%$ BSA for 2 hours at RT followed by DAPI (Pierce Protein Research Products, catalog no. 46290; 1:10,000 in PBS). Tubules were placed on glass slides and mounted in Vectashield (Vector Laboratories). Microscopy was performed with a Leica TCS SP5 confocal microscope with a $\times 40$ oil immersion lens using $543-\mathrm{nm}$ HeNe laser (26\% full power) and 488-nm Ar laser (15\% full power) excitation.

Antibodies for immunofluorescence and immunobistochemistry. Antibodies and titers used were Foxo1 (Cell Signaling Technology, no. 2880; 1:200 immunohistochemistry, 1:50 immunofluorescence), Kit (Cell Signaling Technology, no. 3074; 1:200 immunohistochemistry, 1:50 immunofluorescence), Plzf (1:150 immunohistochemistry, 1:50 immunofluorescence), germ cell nuclear antigen (GCNA; 1:150 immunohistochemistry) (51), Ret (IBL America, no. 18121; 1:20 immunohistochemistry, 1:50 immunofluorescence), p-AKT (Cell Signaling Technology, no. 9271S; 1:100 immunohistochemistry), and Gfro1 (R\&D Systems, catalog no. AF560; 1:100 immunofluorescence).

Germ cell counts and differentiation index. After GCNA immunohistochemistry (see above), testis sections were analyzed by counting $\mathrm{GCNA}^{+}$ cells per tubule ( $n=3$ testes). The average number of $\mathrm{GCNA}^{+}$germ cells per tubule was determined per animal in each genotype. A minimum of 50 tubules was included per genotype of P1 to P7 animals or 75 tubules per genotype of P14 to adult animals. Empty tubules were defined by the complete absence of $\mathrm{GCNA}^{+}$cells. The differentiation index was used as a measure of the percentage of tubules showing multilayer spermatogenesis, defined as the presence of any viable germ cells forming an additional layer not associated with the tubular basement membrane. At least 100 tubules were counted per testis, and testes from at least 2 animals were analyzed for each genotype. Student's 2 -tailed $t$ test was used to assess statistical significance (defined as $P \leq 0.05$ ).

Expression profiling and real-time PCR. cDNA was generated using the High-Capacity cDNA Reverse Transcription Kit (Applied Biosystems, catalog no. 4368814). qPCR was performed as previously described (52) using TaqMan Gene Expression Assays (Applied Biosystems ID numbers, Mm01343388_g1 Dppa4; Mm00490671_m1 Foxo1; Mm00436304_m1 Ret; Mm00456650_m1 Egr2; Mm00842279_g1 Egr4; Mm01240680_m1 Sall4; Mm00521776_m1 Lhx1; Hs99999901_s1 eukaryotic 18s rRNA). Additional information regarding the probe target sequences is available through the Applied Biosystems website (http://www.appliedbiosystems. com) or through the NCBI TaqMan probe database (http://www.ncbi.nlm. nih.gov/projects/genome/probe/doc/ProjTaqMan.shtml). PCR reactions were set up using TaqMan Gene Expression Master Mix (Applied Biosystems, catalog no. 4369016) per the manufacturer's instructions.

Expression profiling. Testes were dissected from P4 mice and immediately homogenized in $1 \mathrm{ml}$ Tripure (Roche). RNA was prepared per the manufacturer's instructions and resuspended in RNase-free water (30 $\mu \mathrm{l} /$ testes). Quality and purity of the RNA was checked by spectrophotometry and gel electrophoresis. RNA samples were submitted to the UTSW Microarray Core for labeling and hybridization per standard protocols. Each genotype was submitted in triplicate using independent testis samples and hybridized to the Illumina Mouse-6 V2 BeadChip. Analysis was performed using BeadStudio software with quantile normalization. Microarray data on mouse Kit expression during postnatal development was obtained from the NCBI, Gene Expression Omnibus Profiles, series GSE640 (53). Microarray data from our study was deposited in the Gene Expression Omnibus (series GSE31282; http://www.ncbi.nlm.nih.gov/projects/geo/ query/acc. cgi? acc $=$ GSE31282).

Statistics. Data were graphed and analyzed using GraphPad Prism 5. Twotailed Student's $t$ tests were used to evaluate significance and calculate $P$ values, with threshold values as described in the Results or figure legends. Error bars represent standard deviation of mean values. A $P$ value of less than 0.05 was considered significant.

\section{Acknowledgments}

We thank Peter Besmer (Sloan-Kettering Institute, New York, New York, USA) for Kit ${ }^{\mathrm{Y} 19 \mathrm{~F}}$ mice, Dario Alessi (MRC Protein Phosphorylation Unit, Dundee, Scotland, United Kingdom) for $P d k 1^{L / L}$ mice, George Enders (University of Kansas Medical Center, Kansas City, Kansas, USA) for the GCNA antibody, and Ned Sharpless, George John, and Wei Guo for technical advice and assistance. This project was supported by award R01HD048690 from the Eunice Kennedy Shriver National Institute Of Child Health and Human Development (NICHD), Award K26RR024196 from the National Center for Research Resources (NCRR), the Lance Armstrong Foundation, and the State of Texas through the Norman Hackerman Advanced Research Program. M.J. Goertz was supported by the UTSW Cell and Molecular Biology training grant 5T32GM008203 from the NIH. The content is solely the responsibility of the authors and does not necessarily represent the official views of the NICHD, NCRR, or the NIH.

Received for publication March 10, 2011, and accepted in revised form July 6, 2011.

Address correspondence to: Diego H. Castrillon, Department of Pathology, University of Texas Southwestern Medical Center, 6000 Harry Hines Boulevard, Dallas, Texas 75390-9072, USA. Phone: 214.648.4032; Fax: 214.648.7355; E-mail: diego.castrillon@ utsouthwestern.edu.
1. Sassone-Corsi P. Unique chromatin remodeling and transcriptional regulation in spermatogenesis. Science. 2002;296(5576):2176-2178.

2. Oatley JM, Brinster RL. Regulation of spermatogonial stem cell self-renewal in mammals. Annu Rev Cell Dev Biol. 2008;24:263-286.

3. Nakagawa T, Sharma M, Nabeshima Y, Braun RE, Yoshida S. Functional hierarchy and reversibility within the murine spermatogenic stem cell compartment. Science. 2010;328(5974):62-67.

4. Nakagawa T, Nabeshima Y, Yoshida S. Functional identification of the actual and potential stem cell compartments in mouse spermatogenesis. Dev Cell. 2007;12(2):195-206.
5. Ohta H, Yomogida K, Dohmae K, Nishimune Y. Regulation of proliferation and differentiation in spermatogonial stem cells: the role of c-kit and its ligand SCF. Development. 2000;127(10):2125-2131.

6 . Hogarth CA, Griswold MD. The key role of vitamin A in spermatogenesis. J Clin Invest. 2010; 120(4):956-962.

7. Meng X, et al. Regulation of cell fate decision of undifferentiated spermatogonia by GDNF. Science. 2000;287(5457):1489-1493.

8. Naughton CK, Jain S, Strickland AM, Gupta A, Milbrandt J. Glial cell-line derived neurotrophic factormediated RET signaling regulates spermatogonial stem cell fate. Biol Reprod. 2006;74(2):314-321.
9. Kanatsu-Shinohara M, et al. Long-term proliferation in culture and germ line transmission of mouse male germ line stem cells. Biol Reprod. 2003; 69(2):612-616.

10. Salih DA, Brunet A. FoxO transcription factors in the maintenance of cellular homeostasis during aging. Curr Opin Cell Biol. 2008;20(2):126-136.

11. Brunet A, et al. Akt promotes cell survival by phosphorylating and inhibiting a Forkhead transcription factor. Cell. 1999;96(6):857-868.

12. Kenyon CJ. The genetics of ageing. Nature. 2010; 464(7288):504-512.

13. Paik JH, et al. FoxOs are lineage-restricted redundant tumor suppressors and regulate endothelial 
cell homeostasis. Cell. 2007;128(2):309-323.

14. Tothova $Z$, et al. FoxOs are critical mediators of hematopoietic stem cell resistance to physiologic oxidative stress. Cell. 2007;128(2):325-339.

15. Renault VM, et al. FoxO3 regulates neural stem cell homeostasis. Cell Stem Cell. 2009;5(5):527-539.

16. Castrillon DH, Miao L, Kollipara R, Horner JW, DePinho RA. Suppression of ovarian follicle activation in mice by the transcription factor Foxo3a. Science. 2003;301(5630):215-218.

17. John GB, Shirley LJ, Gallardo TD, Castrillon DH Specificity of the requirement for Foxo3 in primordial follicle activation. Reproduction. 2007; 133(5):855-863.

18. John GB, Gallardo TD, Shirley LJ, Castrillon DH. Foxo3 is a PI3K-dependent molecular switch controlling the initiation of oocyte growth. Dev Biol. 2008;321(1):197-204

19. Hosaka T, et al. Disruption of forkhead transcription factor (FOXO) family members in mice reveals their functional diversification. Proc Natl Acad Sci U S A. 2004;101(9):2975-2980

20. Gallardo T, Shirley L, John GB, Castrillon DH. Generation of a germ cell-specific mouse transgenic Cre line, Vasa-Cre. Genesis. 2007;45(6):413-417.

21. Buaas FW, et al. Plzf is required in adult male germ cells for stem cell self-renewal. Nat Genet. 2004; 36(6):647-652.

22. Falender AE, et al. Maintenance of spermatogenesis requires TAF4b, a gonad-specific subunit of TFIID Genes Dev. 2005;19(7):794-803.

23. Costoya JA, et al. Essential role of Plzf in maintenance of spermatogonial stem cells. Nat Genet. 2004 36(6):653-659.

24. Kissel $\mathrm{H}$, et al. Point mutation in kit receptor tyrosine kinase reveals essential roles for kit signaling in spermatogenesis and oogenesis without affecting other kit responses. EMBOJ. 2000;19(6):1312-1326.

25. Manova K, Nocka K, Besmer P, Bachvarova RF. Gonadal expression of c-kit encoded at the W locus of the mouse. Development. 1990;110(4):1057-1069.

26. Matson CK, Murphy MW, Griswold MD, Yoshida S, Bardwell VJ, Zarkower D. The mammalian doublesex homolog DMRT1 is a transcriptional gatekeeper that controls the mitosis versus meiosis decision in male germ cells. Dev Cell. 2010;19(4):612-624.

27. Lee J, et al. Akt mediates self-renewal division of mouse spermatogonial stem cells. Development. 2007; 134(10):1853-1859.

28. Lee J, et al. Genetic reconstruction of mouse spermatogonial stem cell self-renewal in vitro by Rascyclin D2 activation. Cell Stem Cell. 2009;5(1):76-86.

29. Kubota H, Avarbock MR, Schmidt JA, Brinster RL. Spermatogonial stem cells derived from infertile $\mathrm{Wv}_{\mathrm{v}} / \mathrm{Wv}_{\mathrm{v}}$ mice self-renew in vitro and generate progeny following transplantation. Biol Reprod. 2009;81(2):293-301.

30. Morimoto $\mathrm{H}$, et al. Phenotypic plasticity of mouse spermatogonial stem cells. PLoS One. 2009;4(11):e7909.

31. Maldonado-Saldivia J, et al. Dppa2 and Dppa4 are closely linked SAP motif genes restricted to pluripotent cells and the germ line. Stem Cells. 2007; 25(1):19-28.

32. Bresnick EH, Lee HY, Fujiwara T, Johnson KD, Keles S. GATA switches as developmental drivers. J Biol Chem. 2010;285(41):31087-31093.

33. Tanaka SS, et al. Loss of Lhx1 activity impacts on the localization of primordial germ cells in the mouse. Dev Dyn. 2010;239(11):2851-2859.

34. Zhang J, et al. Sall4 modulates embryonic stem cell pluripotency and early embryonic development by the transcriptional regulation of Pou5f1. Nat Cell Biol. 2006;8(10):1114-1123.

35. Ollinger R, et al. Deletion of the pluripotency-associated Tex19.1 gene causes activation of endogenous retroviruses and defective spermatogenesis in mice. PLoS Genet. 2008;4(9):e1000199.

36. Tourtellotte WG, Nagarajan R, Auyeung A, Mueller C, Milbrandt J. Infertility associated with incomplete spermatogenic arrest and oligozoospermia in Egr4-deficient mice. Development. 1999; 126(22):5061-5071

37. Hogarth CA, Mitchell D, Small C, Griswold M. EGR4 displays both a cell- and intracellular-specific localization pattern in the developing murine testis. Dev Dyn. 2010;239(11):3106-3114.

38. Oatley JM, Avarbock MR, Brinster RL. Glial cell line-derived neurotrophic factor regulation of genes essential for self-renewal of mouse spermatogonial stem cells is dependent on Src family kinase signaling. J Biol Chem. 2007;282(35):25842-25851.

39. Paik JH, et al. FoxOs cooperatively regulate diverse pathways governing neural stem cell homeostasis. Cell Stem Cell. 2009;5(5):540-553.
40. Oatley JM, Avarbock MR, Telaranta AI, Fearon DT, Brinster RL. Identifying genes important for spermatogonial stem cell self-renewal and survival. Proc Natl Acad Sci U S A. 2006;103(25):9524-9529.

41. Jijiwa $M$, et al. GDNF-mediated signaling via RET tyrosine 1062 is essential for maintenance of spermatogonial stem cells. Genes Cells. 2008;13(4):365-374.

42. Kimura T, et al. Conditional loss of PTEN leads to testicular teratoma and enhances embryonic germ cell production. Development. 2003;130(8):1691-1700.

43. Yilmaz OH, et al. Pten dependence distinguishes haematopoietic stem cells from leukaemia-initiating cells. Nature. 2006;441(7092):475-482.

44. Zhang J, et al. PTEN maintains haematopoietic stem cells and acts in lineage choice and leukaemia prevention. Nature. 2006;441(7092):518-522.

45. Hobbs RM, Seandel M, Falciatori I, Rafii S, Pandolfi PP. Plzf regulates germ line progenitor self-renewal by opposing mTORC1. Cell. 2010;142(3):468-479.

46. Paratcha G, Ledda F. GDNF and GFRalpha: a versatile molecular complex for developing neurons. Trends Neurosci. 2008;31(8):384-391.

47. Carter ME, Brunet A. FOXO transcription factors. Curr Biol. 2007;17(4):R113-R114.

48. Looijenga LH. Human testicular(non)seminomatous germ cell tumours: the clinical implications of recent pathobiological insights. J Pathol. 2009; 218(2):146-162.

49. Li G, et al. Conditional loss of PTEN leads to precocious development and neoplasia in the mammary gland. Development. 2002;129(17):4159-4170.

50. Mora A, et al. Deficiency of PDK1 in cardiac muscle results in heart failure and increased sensitivity to hypoxia. EMBO J. 2003;22(18):4666-4676.

51. Enders GC, MayJJ 2nd. Developmentally regulated expression of a mouse germ cell nuclear antigen examined from embryonic day 11 to adult in male and female mice. Dev Biol. 1994;163(2):331-340.

52. Bookout AL, Cummins CL, Mangelsdorf DJ, Pesola JM, Kramer MF. High-throughput real-time quantitative reverse transcription PCR. Curr Protoc Mol Biol. 2006; Chapter 15:Unit 15.8.

53. Schultz N, Hamra FK, Garbers DL. A multitude of genes expressed solely in meiotic or postmeiotic spermatogenic cells offers a myriad of contraceptive targets. Proc Natl Acad Sci U S A. 2003; 100(21):12201-12206 